\title{
NOTES ON THE RIEMANN ZETA-FUNCTION-IV
}

BY

\section{R.BALASUBRAMANIAN, K.RAMACHANDRA,} A.SANKARANARAYANAN and K.SRINIVAS.

\begin{abstract}
For "Good Dirichlet series" $F(s)$ we prove that there are infinitely many poles $p_{1}+i p_{2}$ in $\Im(s)>C$ for every fixed $C>0$. Also we study the gaps between the numbers $p_{2}$ arranged in the non-decreasing order.

$\S 1$. INTRODUCTION. This paper concerns mainly with some applications of the general result namely Theorem 2 stated in $\S 3$ of [RB, KR, AS, KS] due to R.Balasubramainian, K.Ramachandra, A.Sankaranarayanan and K.Srinivas. (At the outset we would like to point out that their general result can be generalised further as the authors have stated in their paper just refered to). Besides touching about some applications, our main object is to prove the following theorem.
\end{abstract}

THEOREM 1. Let $N$ and $D$ be two finite sets of complex numbers defined by

$$
N \equiv\left\{\alpha_{1}, \alpha_{2} \ldots, \alpha_{k}\right\}, \quad D \equiv\left\{\beta_{1}, \beta_{2}, \ldots, \beta_{l}\right\}
$$

where $0 \leq k \leq l, l \geq 1$ (we stress here that neither the $\alpha$ 's nor the $\beta$ 's need be distinct). Let $P(s)$ be any fixed Dirichlet Polynomial (i.e. a terminating Dirichlet series). Let $F(s)(s=$ $\sigma+i t)$ be defined by

$$
\begin{gathered}
F(s) \equiv\left(P(s) \Pi_{\alpha \epsilon N} \zeta(s+\alpha)\right)\left(\Pi_{\beta \epsilon D} \zeta(s+\beta)\right)^{-1} \\
\equiv F_{1}(s)\left(F_{2}(s)\right)^{-1}
\end{gathered}
$$

say (in an abvious notation). Then under the condition that $F(s)$ is not a terminating Dirichlet series, we have the following results.

拉

1) If either $k<l$ or $\left(k=l\right.$ and $\left.\operatorname{Re}\left(\sum \beta-\sum \alpha\right) \leq 0\right)$, then for every $Y \geq 100$ there exists a pole $p=p_{1}+i p_{2}($ of $F(s))$, depending on $Y$ for which

$$
\left|p_{2}-Y\right| \leq a \log \log Y
$$

\footnotetext{
$\ddagger$ Received on 07-03-2000

Mathematics Subject Classification: 11 M06, 11 M41.

Keywords and Phrases: Poles, Dirichlet series, gaps between poles.
} 
where $a>0$ is a constant independent of $Y$.

(2) If $k=l$ and $\operatorname{Re}\left(\sum \beta-\sum \alpha\right)>0$, then for every $Y \geq 100$ there exists a pole $p=p_{1}+i p_{2}$ (of $F(s))$ depending on $Y$, for which there holds

$$
\left|p_{2}-Y\right| \leq a \log Y
$$

Here $a>0$ is a constant independent of $Y$.

REMARK 1. The theorem is true even when $\zeta(s+\alpha)$ and $\zeta(s+\beta)$ are replaced by $L(s+\alpha)$ and $L(s+\beta)$. (Here $L(s)$ are any L-series with residue class characters).

REMARK 2. The most general application envisaged by the present paper seems to be as follows. Put

$$
F(s)=\frac{\sum P(s) \Pi \zeta_{K_{1}}^{\left(j_{1}\right)}\left(s+\alpha, R_{1}\right)}{\sum Q(s) \Pi \zeta_{K_{2}}^{\left(j_{2}\right)}\left(s+\beta, R_{2}\right)} \equiv \frac{F_{1}(s)}{F_{2}(s)},
$$

where $P(s), Q(s)$ are any fixed Dirichlet polynomials and in the numerator the product is over all possible (finite number of ) quadruplets (with repititions) $\left(K_{1}, j_{1}, R_{1}, \alpha\right)$ varying; $F_{1}(s)$ is an arbitrary sum over all possible products multiplied by $P(s)$, (Also here $\zeta_{K}^{(j)}(s+\alpha, R)(j \geq 0)$ denotes the $j^{\text {th }}$ derivative of the zeta-function of the ray class $R$ in a number field $K)$. The denominator $F_{2}(s)$ is defined in a similar way. One condition that we cannot avoid is that the limit of $F_{2}(\sigma)$ as $\sigma \rightarrow \infty$ should be 1 . The functional equations facilitate the computation of $\phi$ and $\psi$ of the general theorem (see $\S 3$ below), at least in some special cases. We definitely need the condition

$$
0 \leq \max \sum n\left(K_{1}\right) \leq \max \sum n\left(K_{2}\right)
$$

where $n(k)$ denotes the degree of $K$. But the more difficult problem seems to be to guarantee that (at least in some cases) $F(s)$ is a good Dirichlet series. Provided that $\phi$ and $\psi$ satisfy some very generous conditions, we can arive at suitable upper bounds for $\left|p_{2}-Y\right|$. One working method to prove goodness of $F(s)$ is to write

$$
F(s)=\sum_{n=1}^{\infty} a_{n} n^{-s}
$$

and investigate things like $\sum_{p} \lambda(p) a_{p} p^{-s}$ or $\sum \lambda(p) a_{2 p}(2 p)^{-s}(\lambda(p)$ being any complex numbers with absolute value bounded above), with suitable weights which are of the form $p^{-\mu}(\log p)^{\nu}\left(e^{-\frac{p}{2 X}}-e^{-\frac{p}{X}}\right)(X>0$ is a variable and $\mu, \nu$ are real constants $)$.

REMARK 3. As has been remarked there are many applications of the general result. We mention only some. In our Theorem 1 we can replace $\zeta(s+\alpha)$ by any of its derivatives of any 
bounded order. The conclusions will still hold. One special example is $F(s)=\zeta^{\prime \prime}(s)(\zeta(s))^{-1}$. This has infinitely many poles in $0<\sigma<1$ and the gaps between the ordinates are quantified by

$$
\left|p_{2}-Y\right| \leq a \log \log Y .
$$

¿From this we can deduce that if all the zeros of $\zeta(s)$ with ordinates in

$$
Y, Y+a \log \log Y
$$

are simple then for at least one zero $\rho$ (of those just mentioned) we have even $\zeta^{\prime \prime}(\rho) \neq 0$. We may deduce from this other corollaries such as : If all the zeros of $\zeta(s)$ are simple then given any $\delta>0$ there are $\geq Y(a \log \log Y)^{-1}$ zeros $\rho$ in $\left(\left|\sigma-\frac{1}{2}\right| \leq \delta, Y \leq t \leq 2 Y\right)$ with $\zeta^{\prime \prime}(\rho) \neq 0$. This follows by the density theorems for the zeros of $\zeta(s)$.

REMARK 4. Certainly under suitable conditions we can prove that the gaps between the ordinates of the poles of $P(s)(Q(s))^{-1}$ (a quotient of two Dirichlet polynomials) are bounded. One of the very general conditions for this seems to be (apart from $A(\sigma) \rightarrow 1$ as $\sigma \rightarrow \infty$ ) that $P(s)(Q(s))^{-1}$ should be a non-terminating Dirichlet series.

REMARK 5.. If the function $F(s)$ of Theorem 1 is a terminating Dirichlet series then it is necessarily equal to $P(s)$. See Theorem 3 .

$\S 2$. NOTATION. The notation is standard and is self explanatory.

§3. STATEMENT OF THE GENERAL THEOREM. We recall the general theorem from the previous paper (III of this series) for applying it to prove our Theorem 1 in $\S 4$ of the present paper. We state the general theorem as theorem 2 below. We will write $F(s) \equiv F_{1}(s)\left(F_{2}(s)\right)^{-1}$ where $F_{1}(s)$ and $F_{2}(s)$ are explained below and we are interested in the poles of $F(s)$.

In what follows $T$ will exceed a large positive constant. The letters $\phi, \psi$ and $H$ denote positive functions of $T$ and will be bounded below by large positive constants. They are assumed to satisfy

$$
H=o(T), \log \log \phi=O(H)
$$

and

$$
\log \psi=O(H)
$$

where the O-constants are assumed to be sufficiently small.

(A) Let $F_{1}(s)$ and $F_{2}(s)(s=\sigma+i t)$ be two Dirichlet series (which may depend on a parameter $T$ and we consider only the interval $T-H \leq t \leq T+H)$ convergent absolutely in $\sigma \geq C_{0}(\geq$ 
100) and bounded there. The letter $g(>0)$ will denote a large absolute constant and we assume that $F_{1}(s)$ and $F_{2}(s)$ can be continued analytically in $\left.\sigma \geq-g(\log H)(\log \log H)^{-1}\right)$. Also we assume that $F_{2}(\sigma) \rightarrow 1$ as $\sigma \rightarrow \infty$ and that in $\sigma \geq C_{0}$ the function $\left|\log F_{2}(s)\right|$ is bounded above.

(B) Let

$$
\left|F_{2}(s)\right|<\phi^{g} \text { in } \sigma \geq-20 g .
$$

(C) Let $F_{2}(s) \neq 0$ in $-g \geq \sigma \geq-g(\log H)(\log \log H)^{-1}$ and also in the same region we have

$$
\left|F_{1}(s)\left(F_{2}(s)\right)^{-1}\right| \leq C_{4}^{\sigma} \psi \quad\left(C_{4}>0\right),
$$

where $C_{4}$ is a constant. For convenience we assume that the constant $C_{4}$ is bounded below and also above.

(D) Let

$$
\left|F_{1}(s)\right| \leq \exp \left(g^{2}(\log \phi)^{3}\right) \text { in } \sigma \geq-g .
$$

Under these conditions we have

THEOREM 2. (due to R.BALASUBRAMANIAN, K.RAMACHANDRA and A.SANKARANARAYANAN and K.SRINIVAS.) We have a pole $p_{1}+i p_{2}$ of $F(s)$ with

$$
T-H \leq p_{2} \leq T+H,
$$

provided $F(s)$ is good in $\sigma \geq C_{0}$.

§4. APPLICATION TO THE PROOF OF THEOREM 1. All the conditions (for application) are easily verified except that the Dirichlet series (in $\sigma \geq C_{0}$ ) is good ( see the remark at the end of this section). We prove a more general result namely theorem 3 .

THEOREM 3. Suppose that

$$
F(s)=P(s) \Pi_{\alpha}(\zeta(s+\alpha))^{n(\alpha)}
$$

where $\alpha$ runs over a finite (non-empty) set of distinct complex constants and $n(\alpha)$ are nonzero integer constants positive or negative. Also let $P(s)$ be any fixed Dirichlet polynomial which is not identically zero. Then $F(s)$ is a non-terminating Dirichlet series in $\sigma \geq C_{0}$. Moreover $F(s)$ is good in $\sigma \geq C_{0}$ where $C_{0}$ is a large positive constant.

REMARK 1. Note that the condition " $F(s)$ is terminating" is not possible (unless $P(s)$ is identically zero which we have avoided). 
REMARK 2. Our proof will show that similar things about

$$
F(s)=P(s) \Pi_{\alpha, \chi}(L(s+\alpha, \chi))^{n(\alpha, \chi)}
$$

are true (where $L(s, \chi)$ are any L-series with residue class characters) with suitable integer constants $n(\alpha, \chi)$.

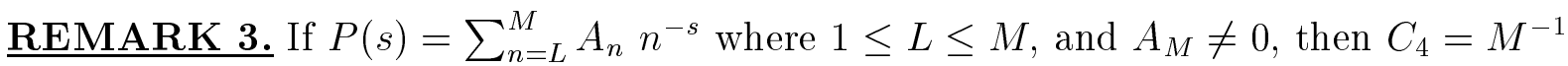
PROOF. Let

$$
P(s)=\sum_{n=L}^{M} A_{n} n^{-s}
$$

where $1 \leq L \leq M$ and $A_{L} \neq 0$. We have (in a notation to be explained presently) in $\sigma \geq C_{0}, F(s)>>>\sum_{p} \sum_{\alpha} n(\alpha) A_{L}(L p)^{-s} p^{-\alpha}$.

(Here as well as in the rest of the proof of theorem 3, by a relation of the type

$$
\sum D_{n}^{(1)} n^{-s}>>>\sum D_{n}^{(2)} n^{-s}
$$

we mean that $\left.\left|D_{n}^{(1)}\right| \gg\left|D_{n}^{(2)}\right|\right)$. It follows that for all $p$ exceeding a large constant $p_{0}$

$$
\sum_{\alpha} n(\alpha) p^{-\alpha}=0
$$

provided $F(s)$ terminates. Let $\alpha_{0}$ be an $\alpha$ for which the real part is the lowest. Then consider the identity

$$
\sum_{\alpha} n(\alpha) p^{-\alpha+\alpha_{0}-1-\delta} \log p=0,(\delta>0),
$$

valid for all primes $p \geq p_{0}$. Summing up over all $p \geq p_{0}$, we have

$$
-\sum n(\alpha) \frac{\zeta^{\prime}\left(\alpha-\alpha_{0}+1+\delta\right)}{\zeta\left(\alpha-\alpha_{0}+1+\delta\right)}=O(1)
$$

the $O(1)$ independent of $\delta$ and comes from the primes $p$ with $2 \leq p \leq p_{0}$. Here all the terms of the sum with $\alpha \neq \alpha_{0}$ are bounded as $\delta \rightarrow 0$ while that with $\alpha=\alpha_{0}$ contributes $n\left(\alpha_{0}\right) \delta^{-1}+O(1)$. This leads to $n\left(\alpha_{0}\right)=0$, a contradiction. This proves the first part of the theorem.

We next proceed to prove the second part. We get back to the relation

$$
F(s)>>>\sum_{p}\left(\sum_{\alpha} n(\alpha) A_{L}(L p)^{-s} p^{-\alpha}\right)
$$


where the sum is over all primes $p$. It suffices to prove that

$$
G(s) \equiv \sum_{p}\left(\sum_{\alpha} n(\alpha) p^{-\alpha+\alpha_{0}} \frac{\log p}{p}\right) p^{-s}
$$

is a good Dirichlet series. Here $\alpha_{0}$ is a certain $\alpha$ with the least real part. For this purpose we consider the function

$$
G(s, X)=\frac{1}{2 \pi i} \int_{A-i \infty}^{A+i \infty} G(s) X^{s}\left(2^{s}-1\right) \Gamma(s) d s
$$

where $A=100\left(1+\sum|\alpha|\right)$. (Note that RHS is nothing but

$$
\sum_{p}\left(\sum_{\alpha} n(\alpha) p^{-\alpha+\alpha_{0}}\right)\left(e^{-\frac{p}{2 X}}-e^{-\frac{p}{X}}\right) \frac{\log p}{p} .
$$

We see easily that $s=0$ is a simple pole of the integrand contributing the residue term $n\left(\alpha_{0}\right)$, which gives (by standard methods of moving the contour)

$$
G(s, X)=n\left(\alpha_{0}\right)+O(\exp (-\sqrt{\log X}))+R
$$

with

$$
R=\sum_{\alpha \neq \alpha_{0}, \operatorname{Re} \alpha=\operatorname{Re} \alpha_{0}} n(\alpha) X^{\alpha_{0}-\alpha}\left(2^{\alpha_{0}-\alpha}-1\right) \Gamma\left(\alpha_{0}-\alpha\right)+O(\exp (-\sqrt{\log X})) .
$$

We now put $X=\exp \left(Y+Y\left(u_{1} \ldots .+u_{r}\right)\right)$ and average the main term in $R$ over the unit cube $0 \leq u_{j} \leq 1(j=1,2, \ldots, r)$. The average is plainly

$$
O\left(\sum 2^{r} Y^{-r}\left|\alpha_{0}-\alpha\right|^{-r}\right)
$$

the sum being as in the main term in $R$. Thus by choosing $r$ to be a large integer, we have

$$
\int G(s, X) d v=n\left(\alpha_{0}\right)+o(1),\left(d v=d u_{1} \ldots d u_{r}\right),
$$

thereby giving

$$
|G(s, X)| \gg 1
$$

for at least one $X$ with $e^{Y} \leq X \leq e^{Y(r+1)}$. This gives what we want after a trival work. Thus theorem 3 is completely proved.

REMARK. For the verification of the conditions on $F(s)$ other than the goodness we can use the functional equation. (For details see our earlier paper II [KR, AS], with the same title). This does not present any serious difficulties. Thus Theorem 1 is completely proved. 
$\S 5$. AN INTERESTING (?) RESULT. Let $a_{0}$ and $b$ be any two complex constants of which $b \neq 0$. Suppose that $a_{0} \zeta^{\prime}(\rho)+b \zeta^{\prime \prime}(\rho)=0$ holds for all zeros $\rho=\beta+i \gamma$ with $Y \leq \gamma \leq Y+a \log \log Y$, where $a>0$ is a certain constant. Then of these zeros there is at least one zero whose order is at least 3 . This follows from our general Theorem.

Finally we wish to include the following corollary to our main theorem.

COROLLARY.Let $\chi_{1}$ and $\chi_{2}$ be two inequivalent proper characters and let $L\left(s, \chi_{1}\right)$ and $L\left(s, \chi_{2}\right)$ be the corresponding L-functions. Put $F(s)=\frac{L\left(s, \chi_{1}\right)}{L\left(s, \chi_{2}\right)}$.

Then there are infinitely many poles of $F(s)$ in $0<\sigma<1, t>0$.

Let $\gamma_{1}, \gamma_{2}, \cdots$ be the ordinates of these poles arranged in the non-decreasing order. Then $\gamma_{n+1}-\gamma_{n} \ll \log \log \gamma_{n}$, whenever $\gamma_{n}>1000$.

The same is also true of the zeros of $F(s)$.

This follows immediately from our main theorem.

\section{R E F E R E N C E S}

[RB, KR, AS, KS], R. BALASUBRAMANIAN, K. RAMACHANDRA, A. SANKARANARAYANAN and K. SRINIVAS, Notes on the Riemann zeta-function-III, HardyRamanujan Journal, Vol. 22, pp. $23-33$.

[KR, AS], K. RAMACHANDRA and A.SANKARANARAYANAN, Notes on the Riemann zeta-function-II, Acta Arithmetica, XCI.4 (1999), pp 351 - 365.

\section{ADDRESS OF THE AUTHORS}

1. R.Balasubramanian

Senior Professor

Matscience

Tharamani P O

Chennai - 600113.

INDIA.

e-mail srini@imsc.ernet.in

2. K.Ramachandra

Hon. Vis. Professor

National Institute of Advanced Studies

Indian Institute of Science Campus

Bangalore-560012

INDIA. 
e-mail kram@math.tifrbng.res.in

3. A.Sankaranarayanan

School of Mathematics

Tata Institute of Fundamental Research

Homi Bhabha Road

MUMBAI-400 005

INDIA.

e-mailsank@math.tifr.res.in

4. K. Srinivas

Matscience

Tharamani P.O.

CHENNAI-600 113

INDIA.

e-mailsrini@imsc.ernet.in

MANUSCRIPT COMPLETED ON 01-03-1999.

revised on 01.10.1999

POST SCRIPT The work in this series of papers will be continued in paper $V$ with the same title (likely in Acta Arithmetica). 